\title{
Forschungsnahes Lernen in studentischen Entwicklungs- und Beraterteams im Modell "Ressource Oriented Sanitation System" der TU Hamburg
}

\section{Ruth Schaldach und Klaus Vosgerau}

Zum Wintersemester 2015/16 wurde an der Technischen Universität Hamburg (TUHH) das Modul „Resources Oriented Sanitation Systems“ didaktisch neu im Sinne des Forschenden Lernens (Huber, 2009) konzipiert und in dem Semester als Pilotprojekt durchgeführt. Seitdem setzen sich darin turnusmäßig rund 40 Masterstudierende aus verschiedenen Studiengängen sowie internationale Austauschstudierende aktiv mit einem aktuellen Schwerpunkt der Forschung und Entwicklung am Institut für Abwasserwirtschaft und Gewässerschutz (AWW) und mit Forschungsergebnissen angrenzender Fachgebiete auseinander.

\section{$1 \quad$ Welche Lernziele sollen mit dem neuen Modul erreicht werden?}

Ziel des neu gestalteten Moduls ist, die Studierenden zu befähigen, als Umweltingenieurinnen und -ingenieure selbstständig zur nachhaltigen ländlichen Entwicklung unter verschiedenartigen klimatischen Bedingungen beizutragen. Eingeübt wird dies am Beispiel der Entwicklung und Umsetzung von Trockentoilettensystemen in der gemäßigten Klimazone Norddeutschlands. Dadurch sollen die Lernenden in die Lage versetzt werden, die Notwendigkeit eines Wandels der Ressourcenkreisläufe nicht nur auf theoretischer und globaler Ebene mithilfe allgemeiner fachwissen- 
schaftlicher Argumentationen begründen, sondern auch exemplarisch, praktisch und nachhaltig dazu beitragen zu können.

Um diese Ziele zu erreichen, wurde ein bestehendes Lernmodul durch eine Kombination von Vorlesungen, Präsentationen, Diskussionen und semesterbegleitender Projektarbeit neu ausgestaltet. Dabei lösen studentische Projektgruppen selbstgesteuert und wissenschaftsbasiert reale Probleme im Fachgebiet „ressourcenorientierte Sanitärsysteme" des Umweltingenieurwesens. Zur Problembearbeitung müssen gesellschaftliche, wirtschaftliche, rechtliche, technische und gestalterische Aspekte einbezogen und miteinander verknüpft werden. Die Teams untersuchen Toilettensysteme, die in verschiedene Nutzungskontexte eingebettet sind: ein öffentliches Trockentoilettenpilotprojekt, eine Kleingartentoilette sowie die Toilettenanlagen eines Musikfestivals, zweier Bauwagenplätze und eines Permakulturgarten-Projektes.

Mit jedem dieser Systeme ist ein Verbesserungsbedarf verbunden, der von den Studierenden in eine Problemdefinition übersetzt wird. Auch wenn alle Fälle das Untersuchungsobjekt der Trockentoilette als Teilaspekt des EcoSan (Ecological Sanitation)-Ansatzes (Lange \& Otterpohl, 2000) gemein haben, unterscheiden sich damit verknüpfte, differenzierende Parameter stark voneinander: $\mathrm{Zu}$ den Problemdimensionen gehören zum Beispiel der bauliche Zustand, der Grad der Ausgereiftheit der bisherigen Umsetzung sowie die Anforderungen der Nutzerinnen und Nutzer. Der genaue Charakter eines jeweiligen Problems unterscheidet sich von Fall zu Fall. Die Systeme divergieren nicht nur baulich, sondern sind auch in unterschiedliche räumliche sowie sozioökonomische Kontexte eingebettet.

Ausgehend von ihrer Problemdefinition entwickeln die Studierenden selbstständig ihre Untersuchungsfragen, was durch erste Begehungen und Informationsgespräche unterstützt wird. Je nach entwickelter Fragestellung werden dann individuelle Untersuchungspläne entworfen. Daran schließt sich die Analyse der jeweiligen Sanitärsysteme mit einem multidisziplinären Instrumentarium an (siehe Abb. 1). Die Studierenden wählen verschiedene Methoden zur Datensammlung und -analyse aus und wenden sie an; das Spektrum reicht von der Interviewdurchführung bis zur biologisch-chemischen Laboranalytik. Nach der Konzeptentwicklung in der Gruppe werden die neuen Ideen präsentiert und in Teilbereichen mit den Nutzerinden umgesetzt. Ziel ist schließlich, die Betreibenden der Anlagen in die Lage zu versetzen, ihre Systeme selbstständig und kontinuierlich durch weitere Verbesserungsmaßnahmen zu optimieren. 


\section{Welcher didaktische Ansatz zur Integration von Forschung in die Lehre wurde gewählt?}

Das Lehrkonzept stellt vier der von Huber entwickelten Kernelemente des forschenden Lernens ins Zentrum, welches er als Lernen im und durch den Forschungsprozess versteht (Huber, 2004, 2009): die selbstständige Eingrenzung und Definition der Problemstellung, die eigenständige Wahl der Methodik, die Analyse und Interpretation der Daten mit kritischer Einbettung in die aktuelle Fachdiskussion sowie die eigenständige Organisation des Ablaufs von Forschung und Entwicklung als Ausgestaltung der sozialen Prozessebene. Konkretisiert wird dieses Rahmenkonzept durch die Methodik des problem- und projektbasierten Lernens. Dabei grenzen die Studierenden die Problemstellung im Vorfeld der eigenständigen Problemdefinition mithilfe der modellhaften Schrittfolge des problembasierten Lernens ( $\mathrm{PbBL}$ ) ein; die übergreifende Strukturierung des Lern- und Forschungsprozesses geschieht hingegen im Sinne des projektbasierten Lernens $(\mathrm{PjBL})$, wobei aufeinander folgende Lern- und Arbeitseinheiten von Gruppen als Teilschritte in einem Projektablauf strukturiert und organisiert werden (Braasch \& Simon, 2014).

Das problembezogene Setting bewirkt durch die direkte Anwendbarkeit der Resultate auf zweierlei Weise bereits von Beginn an ein hohes Erkenntnisinteresse bei den Lernenden: (1) Es besteht ein direkter Bezug zu den Nutznießenden der Ergebnisse, sodass die konkrete, bedürfnisbezogene Problemlösung deutlich erfahrbar wird. (2) Die Übertragbarkeit der Resultate spielt eine große Rolle für Interesse und Motivation, denn die Fortschritte in den studentischen Forschungsund Entwicklungsprojekten des Moduls führen nicht nur zu Verbesserungen in den Einzelfällen, sondern tragen zugleich beispielhaft und erprobend zu Lösungskonzepten für drängende globale Sanitärfragen bei. Damit wird indirekt auch ein emanzipatorisches Erkenntnis- und Lösungsinteresse wirksam und motiviert das individuelle Lernhandeln.

Ingenieurwissenschaft kann in dem neuen Modul auf mehreren Ebenen als sozialer Prozess erfahren werden: (1) Die Studierenden durchlaufen in einer für die Ingenieurwissenschaften typischen Weise den Forschungsprozess, nehmen dabei immer wieder eigene Entscheidungen vor und organisieren ihre Arbeit in einem vorgegebenen, groben Rahmen (siehe Abb. 1) selbstständig. (2) Das Lernen in den Projekten ist durch eine enge Zusammenarbeit mit den „Auftraggebern“ gekennzeichnet, die ein direktes Interesse daran haben, neue, durch Forschung gewonnene Erkenntnisse zu begleiten und zu unterstützen. (3) Es wird in multidisziplinären und interkulturellen Gruppen gearbeitet, die sich gegenseitig konstruktives Feedback geben und die Projektphasen gemeinsam durchlaufen. 


\section{Welcher Rahmen wird dem Ablauf des studentischen Arbeitsprozesses gegeben?}

Nachdem die Studierenden in einer Vorlesung in den Themenkomplex eingeführt wurden, werden die Studierendenteams nach vorab festgelegten Parametern zusammengestellt. Der Auswahlprozess basiert auf Kurzbewerbungen in Form strukturierter, von den Studierenden zur Selbstauskunft angefertigter Profile, die beispielsweise über vorliegende Arbeitserfahrungen, Studienschwerpunkte, aber auch außercurriculare Aktivitäten mit Relevanz zu den Projekten Auskunft geben können. Das Ziel ist dabei, eine möglichst diverse - das heißt vor allem multidisziplinäre und internationale - Zusammensetzung zu erhalten, damit in jeder Gruppe ein breit gefächerter Wissenspool und ein möglichst großer Erfahrungshorizont gegeben sind. Zusätzlich wird festgelegt, dass in jeder Gruppe eine Person teilnimmt, die über deutsche Sprachkenntnisse verfügt, um die Kommunikation mit den Fallgebern zu erleichtern.

Um den wissenschaftlichen Austausch und Arbeitsprozess zu unterstützen, wurde ein minimaler, allgemein verbindlicher Projektrahmen erstellt, der es ermöglicht, die Lernziele im Verlauf eines Semesters zu erreichen. Dafür wurden drei Phasen definiert und Feedbackschleifen eingeplant (siehe Abb. 1).

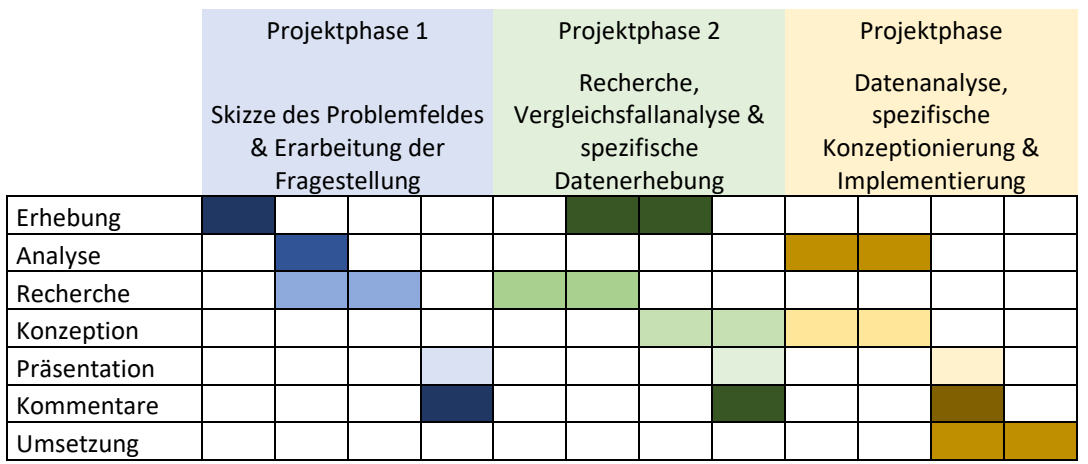

Abb. 1 Projektphasen

Mittels dieser verbindlichen Projektphasen wird der Prozess des Forschens im Verständnis des Forschenden Lernens (Huber, 2009) so operationalisiert, dass die Arbeitsflüsse der verschiedenen Projektgruppen grundsätzlich synchronisiert sind. 
Dies ermöglicht es, im Semesterablauf ungefähr zur selben Zeit Themen von gemeinsamem Interesse zu diskutieren, Peer-Feedback zu geben und zu erhalten und auch gezielten Input zu übergreifenden Problemen durch externe Fachleute anzubieten.

Zur Bearbeitung der Problemstellungen wird in der Vorlesung ein breit gefächertes Analyseinstrumentarium vermittelt. Die schließlich von den Studierenden angewendeten Maßnahmen bauen auf den vorgestellten Methoden auf und müssen selbstständig recherchiert und ausgewählt werden.

Die Präsentationstermine bilden den Abschluss einer Projektphase und die Basis für die nächste. Das studentische Peer-Feedback findet in einem begleitenden Projektseminar in Form von Kommentaren statt. Mitstudierende aus anderen Gruppen stellen sich vorab als Kommentierende zur Verfügung und erhalten vor den Präsentationen das Material, um einen eigens durch Recherche fundierten Kommentar vorzubereiten und zu referieren. Es folgen dann noch Kommentare von Fachleuten (auch auswärtigen, darunter auch Anwendende aus der Praxis), woraufhin die Diskussion schließlich geöffnet wird. Dieses forschungsäquivalente Vorgehen ermöglicht die Entwicklung der Kompetenz, in einen wissenschaftlichen Austausch zu treten, konstruktive Kritik zu üben und von dieser selbst profitieren zu können.

\section{$4 \quad$ Reflexion von erster Durchführung und Konzept}

Die Veranstaltung wird in dreierlei Hinsicht evaluiert:

1. TAP (Teaching Analysis Poll) Gruppendiskussionsverfahren, durchgeführt in der Mitte des Semesters;

2. Feedbackgespräch mit den Studierenden und dem Lehrendenteam am Ende des Semesters;

3. CheckIng - schriftliches, anonymisiertes Evaluationsverfahren der TUHH, das nach jeder Veranstaltung durchgeführt wird.

Kritisiert wird von einigen Studierenden der Umfang des Arbeitsaufwandes; Nachfragen ergaben, dass dieser in manchen Gruppen aufgrund interner Kommunikationsprobleme grösser war als in anderen Gruppen. Der Zeitaufwand des Auswahlprozesses wird ebenso kritisiert wie das Konzept, eine temporäre Teilgruppe der Studierenden maßgeblich über die Zusammensetzung aller Projektgruppen entscheiden zu lassen. Es stellte sich als große Anforderung heraus, hierbei die nötige Distanz zu wahren, da die Studierenden in einer Doppelrolle sind, bei der die Diversitätskriterien persönlichen Vorlieben gegenüberstehen. 
Die Gesamtevaluation zeigt, dass die forschungsbezogenen Studienprojekte mit realer Problemstellung, selbstständiger Erarbeitung technischer und planerischer Lösungen und individueller Beratung verschiedener Nutzungsgruppen als sehr motivierend empfunden werden. Zugleich sehen die Studierenden sich besser befähigt, auch in Zukunft Projekte eigenständig und forschungsnah umzusetzen. Von den Studierenden wird die gute Diskussionsatmosphäre gelobt, die aufgrund der respektvollen und konstruktiv vorgetragenen Kommentare seitens der Peer-Gruppe und der Fachleute entsteht. Als besonders wertvoll wird angesehen, einüben zu können, konstruktive Kritik zu geben.

Aus Sicht der Lehrenden erweist sich das Konzept der Projektphasenpräsentationen als sehr hilfreich, da es sowohl ihnen als auch den Mitstudierenden ermöglicht, punktgenau und zielführend Feedback zu geben. Als besonders wertvoll wird dabei von allen Beteiligten das Peer-Feedback in Form von Kommentaren angesehen. Die mündlich vorgetragenen und dann verschriftlichten Kommentare ermöglichen einen intensiven Austausch zwischen den Entwicklungsgruppen und tragen nach allgemeiner Auffassung zur Steigerung der Qualität der Projektergebnisse bei.

Das forschungsnahe Lernen im neugestalteten Modul ermöglicht es den Studierenden, ihre entwickelten Prozesskompetenzen später auf Problemlösungen in anderen Zusammenhängen zu übertragen, zum Beispiel unter anderen klimatischen Bedingungen und neuen soziokulturellen Verhältnissen. Dabei können die Lernenden von der Lernerfahrung profitieren, die theoretisch begründete Notwendigkeit eines Wandels der Ressourcenkreisläufe lokal, exemplarisch und wissenschaftsgeleitet umzusetzen.

\section{Literatur}

Braasch, M. \& Simon, S. (2014). Problembasiertes Lernen (PBL). Hamburg: Zentrum für Lehre und Lernen (ZLL) der Technischen Universität Hamburg. Verfügbar unter https:// www2.tuhh.de/zll/leistungen/pbl-problem-based-learning [30.11.2018].

Huber, L. (2004). Forschendes Lernen: 10 Thesen zum Verhältnis von Forschung und Lehre aus der Perspektive des Studiums. Die Hochschule: Journal für Wissenschaft und Bildung, 2, S. 29-49.

Huber, L. (2009). Warum Forschendes Lernen nötig und möglich ist. In L. Huber, J. Hellmer \& F. Schneider (Hrsg.), Forschendes Lernen im Studium: Aktuelle Konzepte und Erfahrungen (Bd. 10, S.9-35). Bielefeld: UVW.

Lange, J. \& Otterpohl, R. (2000). Abwasser: Handbuch zu einer zukunftsfähigen Wasserwirtschaft (Ökologie aktuell, 2. Aufl.). Donaueschingen-Pfohren: Mall-Beton-Verlags. 


\section{Zur Autorin und zum Autor}

Dr. Ruth Schaldach studierte an der Macquarie University Sydney und der Universität Hamburg und erlangte einen Master in internationalen Beziehungen und europäischen Studien. Seit Anfang 2017 leitet sie das Online-Lehrprojekt RUVIVAL an der Technischen Universität Hamburg und führt seit 2015 verschiedene Lehrinnovationsprojekte am Institut für Abwasserwirtschaft und Gewässerschutz (AWW) durch. E-Mail: ruth.schaldach@tuhh.de

Dr. Klaus Vosgerau, Dipl.-Ing., studierte Sozialwissenschaften und Stadtplanung an den Universitäten Kassel und Oldenburg und forschte danach zur Sozialisation in Hochschule und Stadt. Seit 2005 ist er in der Beratung und Entwicklung von Hochschulen aktiv, zunächst bei der Bund-Länder-Einrichtung HIS in Hannover, ab 2010 als Geschäftsführer des Promotionszentrums der Universität Bremen und seit 2012 als Projektkoordinator am Zentrum für Lehre und Lernen der Technischen Universität Hamburg. E-Mail: klaus.vosgerau@tuhh.de

Open Access Dieses Kapitel wird unter der Creative Commons Namensnennung 4.0 International Lizenz (http://creativecommons.org/licenses/by/4.0/deed.de) veröffentlicht, welche die Nutzung, Vervielfältigung, Bearbeitung, Verbreitung und Wiedergabe in jeglichem Medium und Format erlaubt, sofern Sie den/die ursprünglichen Autor(en) und die Quelle ordnungsgemäß nennen, einen Link zur Creative Commons Lizenz beifügen und angeben, ob Änderungen vorgenommen wurden.

Die in diesem Kapitel enthaltenen Bilder und sonstiges Drittmaterial unterliegen ebenfalls der genannten Creative Commons Lizenz, sofern sich aus der Abbildungslegende nichts anderes ergibt. Sofern das betreffende Material nicht unter der genannten Creative Commons Lizenz steht und die betreffende Handlung nicht nach gesetzlichen Vorschriften erlaubt ist, ist für die oben aufgeführten Weiterverwendungen des Materials die Einwilligung des jeweiligen Rechteinhabers einzuholen.

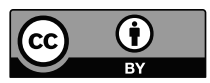

